\title{
Imaging of deep venous thrombosis using radioactive-labeled tirofiban
}

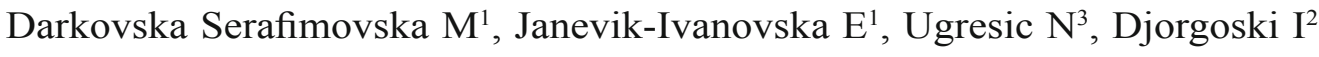 \\ Goce Delcev University, Faculty of Medical Sciences, Stip, Republic of Macedonia. \\ emilija.janevik@ugd.edu.mk
}

\begin{abstract}
The development of radiolabeled small peptide or peptidomimetic ligands can bind platelets and their specific expressed receptor have been suggested as a new approach to detect the clot location and, more essentially, to determine the age and morphology of the evolving thrombus. This new approach is focused on the use of a series of radiolabeled platelet GPIIb/IIla receptor antagonists.

Tirofiban N-(butylsulfonyl)- 4-O-(4-(4-piperidyl)-L-tyrosine is a non-peptide tyrosine derivate. The aim of the study was to introduce radioactive-labeled tirofiban as a specific imaging agent for acute DVT.

The labeling was performed with technetium-99 in the presence of a stannous reducing agent. The labeled preparation showed fast blood clearance in a normal rat model (without induced thrombosis). More than $80 \%$ of the injected dose was eliminated from the circulation in the first hour after injection. Biodistribution and visualization of the labeled molecule was carried out using an experimental model of thrombosis in a male Wistar rat. Planar images were obtained 30 and $60 \mathrm{~min}$ after application of $2 \times 10^{6} \mathrm{imp} / \mathrm{min} 99 \mathrm{~m}$-technetium-tirofiban in the rat's tail vein. Sensitivity and specificity were determined using the ratio of 'left leg positive for DVT' to 'right leg negative for DVT'. The obtained ratio was 1.54 after $30 \mathrm{~min}$ and 5.04 after $60 \mathrm{~min}$. These values were considered positive in the detection of acute DVT. The high DVT uptake shows that radiolabeled tirofiban in the introduced rat model can be a promising agent for imaging the deep venous thrombosis (Fig. 7, Ref. 25). Text in PDF www.elis.sk.

KEY WORDS: radionuclide labeling, imaging, deep venous thrombosis; 99mTc-tirofiban, rat model.
\end{abstract}

\section{Introduction}

Venous thromboembolism is a complex vascular disease with a multifactorial pathogenesis that results in significant morbidity and mortality. Its first manifestation is deep venous thrombosis (DVT), which is most common and usually arises in the deep veins of the calf and spreads upwards. Pulmonary embolism is the second manifestation, which is more serious and occurs as a complication of DVT proximal to the deep calf veins (1).

Despite the remarkably high incidence of deep venous thrombosis (DVT) and pulmonary embolism (PE) in high-risk populations, hospitalized patients, and general population, the accuracy of the diagnoses of DVT and PE continue to be unreliable $(2,3)$.

All available imaging procedures (duplex ultrasound, magnetic resonance and contrast venography as a standard test for validating new diagnostic procedures) do not reflect the metabolic activity of the clot and therefore they may overestimate the presence of active clots (4). Only nuclear medical examinations can provide an

${ }^{1}$ Goce Delcev University, Faculty of Medical Sciences, Stip, Republic of Macedonia, ${ }^{2}$ University St. Cyril and Methodius, Faculty of Natural Science and Mathematics, Skopje, Republic of Macedonia, and ${ }^{3}$ University of Belgrade, Faculty of Pharmacy, Belgrade, Serbia

Address for correspondence: E. Janevic Ivanovska, University Goce Delcev, Faculty of Medical Sciences, Krste Misirkov bb, 2000 Stip, Republic of Macedonia. image that includes the information on thrombus formation (25). The development of radioactive agents for scintigraphic imaging of DVT and PE is centered on the use of $99 \mathrm{mTc}$-labeled peptides or peptidomimetics specific for resting or activated platelets (49). They are smaller in size, their production is easier than that of monoclonal antibodies, their clearance from circulation is expected to be more rapid than that of radiolabeled proteins, and they are less likely to induce any immunologic reaction. At the same time, in most cases, their receptor specificity and binding constants are as high as those of the monoclonal antibodies.

The $99 \mathrm{~m}$-technetium is a radionuclide that is inexpensive, easy to obtain worldwide, and it decays with emission of $\gamma$-rays $(140 \mathrm{keV}, 90 \%)$ that can be efficiently detected by $\gamma$-cameras. Its half-life $(6 \mathrm{~h})$ is long enough to perform examinations before excessive radioactive decay occurs, yet not too long to persist in the body long after the examinations are performed.

Our approach was focused on the use of a series of radiolabeled platelet GPIIb/IIIa receptor antagonists $(6,10)$. The GPIIb/IIIa receptors are expressed on the membrane surface of activated platelets and play an integral role in platelet aggregation and thrombus formation (10). Initial actions in thrombus formation frequently involve the activation of platelets by thrombogenic conditions and their subsequent aggregation. Platelet aggregation is mediated by fibrinogen which binds via the Arg-Gly-Asp (RGD) sequence to the GPIIb/IIIa receptor expressed on activated platelets. Since the 


\section{1-626}

GPIIb/IIIa receptors are expressed only on the membrane surface of activated platelets, with 50 000-90 $000 \mathrm{GPIIb} / \mathrm{III}$ binding sites per platelet (2), the GPIIb/IIIa receptor makes an excellent target for the development of an imaging agent bound with high specificity to activated rather than to inactive platelets. They would be differentially incorporated in the thrombus (activated platelets) and the circulating platelets (resting or relatively less activated) $(11,12)$.

These molecules represent glycoprotein (GPIIb/IIIa) receptor antagonists and they act as true biochemical markers of active thrombosis $(10,11)$.

One peptide, 99mTc-P280 (5), is approved by the Food and Drug Administration under the trade name AcuTect (99mTc-P280; Diatide, Inc.) (8), which can image acute thrombi but not old clots or PE.

The goal of our work was to use a small non-peptide derivate or peptidomimetic ligands with high specificity for the GPIIb/IIIa receptor and incorporate a convenient radionuclide for imaging purposes was initiated from the already existing data presented using labeled peptides and GPIIb/IIIa receptor antagonists in the diagnosis of acute DVT. One promising GPIIb/IIIa receptor antagonist is tirofiban (AggrastatTM, Merck, Inc.,), a nonpeptide tyrosine derivate that inhibits fibrinogen binding (14) Tirofiban hydrochloride monohydrate is chemically described as $\mathrm{N}$-(butylsulfonyl)-O-(4-(4-piperidinyl)butyl)-L-tyrosine monohydrochloride monohydrate (Fig. 1). The empirical formula of tirofiban is $\mathrm{C}_{22} \mathrm{H}_{36} \mathrm{~N}_{2} \mathrm{O}_{5} \mathrm{~S} \cdot \mathrm{HCl} \cdot \mathrm{H}_{2} \mathrm{O}$ and its molecular weight of $495.08(2,3)$.
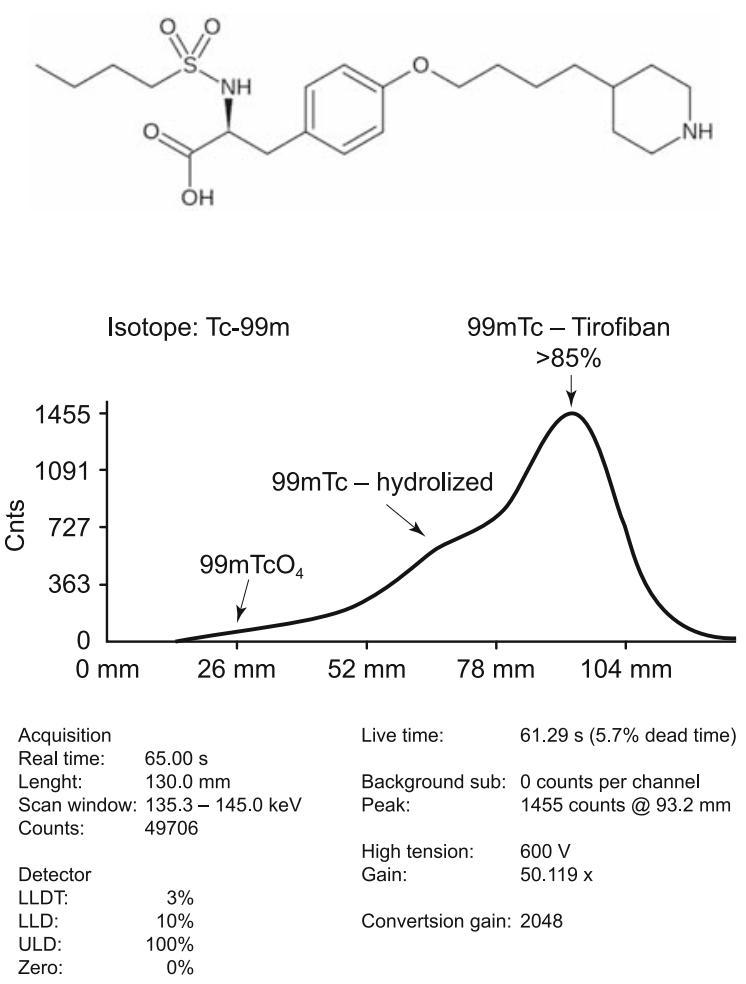

Fig. 1. Chemical structure of Tirofiban hydrochloride.
It is useful in combination with heparin and aspirin in the management of patients with unstable angina or non-Q-wave myocardial infarction, including patients who may subsequently undergo percutaneous transluminal coronary angioplasty (PTCA0 $(13,14)$.

Adjunctive therapy with a GP IIb/IIIa-receptor inhibitor can reduce the incidence of cardiac ischemic events, including subsequent myocardial infarction (MI) and death, in patients with nonST-segment-elevation acute coronary syndromes $(5,6)$.

When tirofiban is prescribed and administered to the patient according to the recommended treatment, $>90 \%$ inhibition is attained by the end of the 30-minute infusion. Platelet aggregation inhibition is reversible following cessation of the infusion of tirofiban. Tirofiban with a half-life of approximately 2 hours (15) is not strongly bound to plasma protein, and protein binding is concentration-independent in the range of $0.01-25 \mu \mathrm{g} \mathrm{mL}^{-1}$. The unbound fraction in human plasma is $35 \%$. The distribution volume of tirofiban in the steady state is about 30 liters.

To obtain the concentration of tirofiban appropriate for labeling as a potential imaging radiopharmaceutical and at the same time in order not to receive its therapeutic effect, we have labeled tirofiban with $99 \mathrm{~m}$-technetium and evaluated it in vitro and in rats with experimental venous thrombosis established and modified afterwards according to the needs of a particular line of research $(16,17)$.

\section{Material and methods}

Materials

Tirofiban

The structure of tirofiban (tirofiban hydrochloride monohydrate) N- (butylsulfonyl)-O-(4-(4-piperidinyl)butyl)-L-tyrosine monohydrochloride monohydrate is shown in Figure 1. Tirofiban hydrochloride monohydrate is a white to off-white, non-hygroscopic, free-flowing powder, with a molecular weight of 495.08. It is very slightly soluble in water $(2,3)$.

Isotope-99m-technetium

The 99m-technetium-pertechnetate was obtained from a commercial 99Mo/ 99mTc generator (10 GBq) (Schering /CIS-biointernational).

\section{Radiolabeling}

Labeling of tirofiban with 99m-technetium

Tirofiban was labeled using the method of direct labeling under nitrogen. The kit freeze dried formulation contains a mixture of tirofiban (20 nmol) dissolved in buffer (ethanol 96\%/PBS 0.01 $\mathrm{M}, \mathrm{pH} 7.3,1: 3 \mathrm{vol} . / \mathrm{vol}$.) and stannous chloride $(10 \mathrm{nmol})$ as a reducing agent. Sodium $(99 \mathrm{mTc})$ perthechnetate (specific activity $740-4500 \mathrm{MBq} / \mathrm{mL}$ ) containing $100 \mathrm{MBq} / \mathrm{mL}$ was added and the reaction mixture incubated for $15 \mathrm{~min}$ at room temperature.

The quality control was done by paper chromatography and instant thin layer chromatography (ITLC) using two solvents, namely $95 \%$ acetone and saline. The percentage of labeled yield was recorded by gamma scanner (Veenstra Instrumenten B.V. VCS-103 V1.06).

\section{Blood clearance}

The 99m-technetium-tirofiban was injected intravenously into 
rats. Blood samples were drawn from the previously prepared carotid vein using a sterile syringe at $5,15,30,45 \mathrm{~min}$ and $1,2,4$, 6 and $24 \mathrm{~h}$ after injection. All samples were of the same volume and their radioactivity was measured in a gamma counter and compared with the standard.

In vitro platelet binding

The binding study was performed with rat and human platelets isolated and treated according to protocol for platelet labeling (TROMBO-SCINT). Platelets were incubated for $30 \mathrm{~min}$ with tirofiban radiolabeled with $99 \mathrm{~m}$-technetium. The percentage of binding was measured after one and two washing steps.

Animal studies

Male Wistar rats weighing 220-250 g were used throughout the study, anaesthetized by intraperitoneal injection of water solution of Nesdonal (concentration $20 \mathrm{mg} / \mathrm{kg}$ body weight).

\section{Experimental animal model $(18,19)$}

Venous thrombosis was induced by ligature of the femoral vein in rats whose blood was made hypercoagulable by intravenous administration of tissue thrombin (17). For the thrombosis model, the body temperature of rats was maintained at $37^{\circ} \mathrm{C}$. In brief, a short incision was made in the skin and subcutaneous tissue in the left groin region and the femoral neurovascular sheath was gently exposed (20). An approximately 10-mm long portion of the left femoral vein, distal to the inguinal ligament was isolated by rubbing it against the blade of a pair of forceps, and this segment collapsed. The collapsed segment of femoral vein between the clamps was traumatized by striking, and twenty units of thrombin (in $0.2 \mathrm{~mL}$ saline) were injected into the segment with a needle. A semiconstricting ligature was placed downstream to prevent the clot from slipping away.

Imaging

The thrombi developed in the rats were visualized using tirofiban radiolabeled with $99 \mathrm{~m}$-technetium. Planar images were obtained 30 and $60 \mathrm{~min}$ after the application of $2 \times 10^{6}$ counts/ $\min$ in $50-100 \mu \mathrm{L} 99 \mathrm{~m}$-technetium-tirofiban in the rat's tail vein. Syringes should be measured before and after injection in order to determine accurately the radioactivity of material injected. The

Isotope: Tc-99m

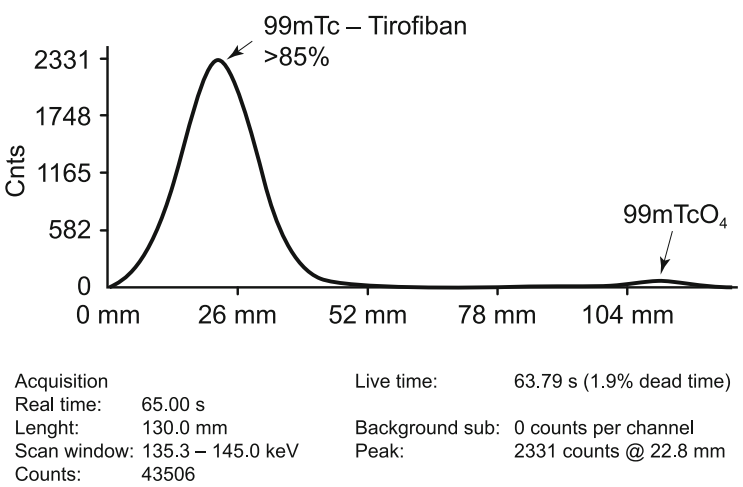

Fig. 2. ITLC radiochromatograms of $99 \mathrm{mTc}$-tirofiban in (a) $95 \%$ acetone (b) saline.

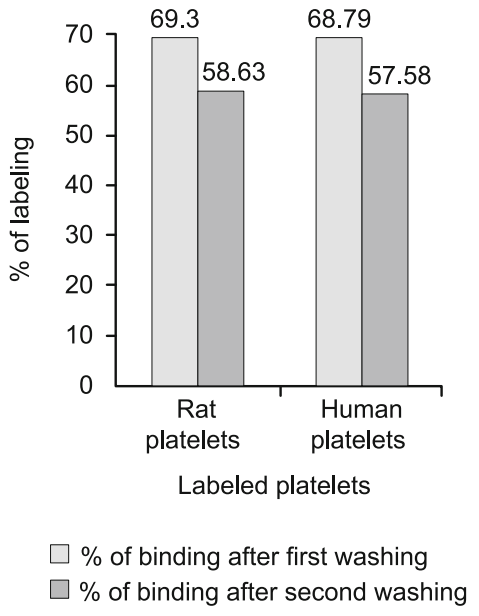

Fig. 3. In vitro binding of rat and human platelets isolated and treated with radiolabeled tirofiban.

sensitivity and specificity of the radiopharmaceuticals were determined using the ratio of 'left leg positive for DVT' to 'right leg negative for DVT' using the ROI technique.

\section{Biodistribution}

The biodistribution studies were carried out using the same experimental model of thrombosis in male Wistar rat and injecting the radiolabeled products. After the desired time period had elapsed, the animals were sacrificed and the samples of organs of interest (heart, lung, liver, spleen, kidney, thrombotic and normal tissue) were collected and placed in pre-weighed counting tubes. These were then counted in a gamma counter together with the standard prepared from a known dilution of injected material (preferably prepared at the time of injection). The total activity injected into each rat was determined and the activity remaining in the tail substracted. The uptake of labeled products in each tissue was calculated and the specific uptake of the products quantified using the ratio of 'left leg positive for DVT' to 'right leg negative for DVT'.

\section{Results}

This study used a rat model of DVT to evaluate radiolabeled tirofiban platelets GPIIb/IIIa antagonist for its potential use in the detection of rapidly growing venous thrombi. Two different methods of labeling were selected using $99 \mathrm{~m}$-technetium as radioisotope.

Labelling of tirofiban with $99 \mathrm{~m}$-technetium

The specific activity of the radiolabelled product was $9.2 \times$ 1018 to $1.0 \times 1019$ counts $/ \mathrm{min} / \mathrm{mol}$.

The percentage of obtained complex after labeling was more than $95 \%$ (in acetone), more than $85 \%$ (in saline), with free pertechnetate (99mTcO4 -) less than $5 \%$ (Fig. 2).

The labeled product was stable without changing the percentage of labeling after $2 \mathrm{~h}$ at room temperature.

After determination of radiochemical purity, it was concluded that the product could be tested for in vitro binding using normal platelets from humans and rats. 


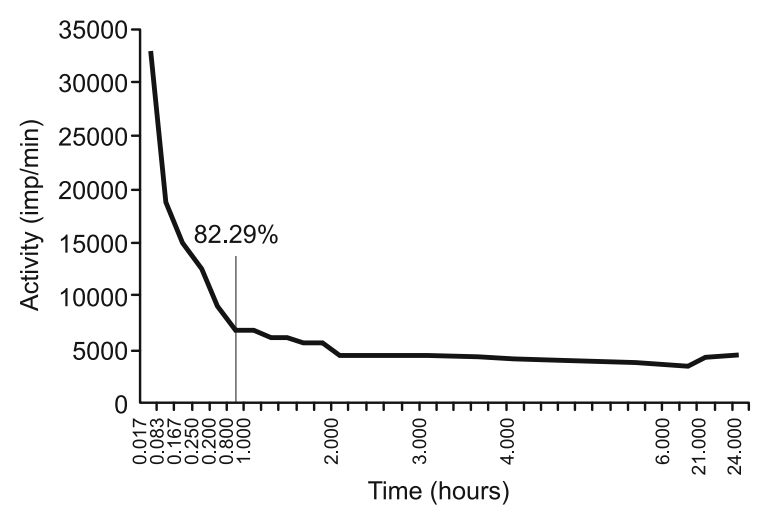

Fig. 4. Blood clearance of radiolabeled tirofiban - 99mTc-tirofiban in normal rat.

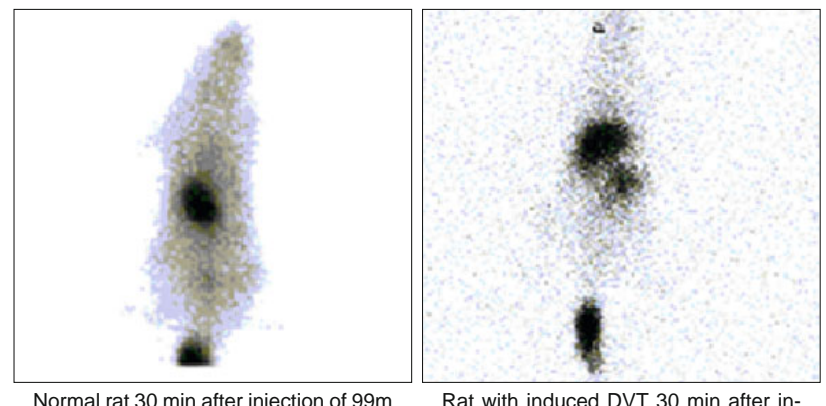

Technetium - Tirofiban $\quad$ jection of $99 \mathrm{~m}$ Technetium - Tirofiban

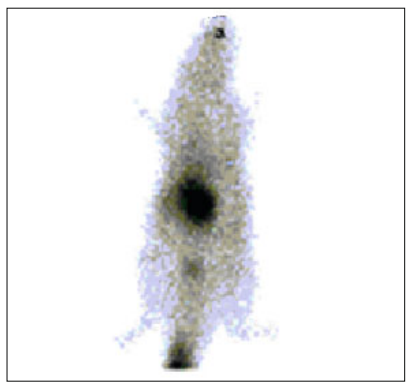

Normal rat 60 min after injection of $99 \mathrm{~m}$ Technetium - Tirofiban

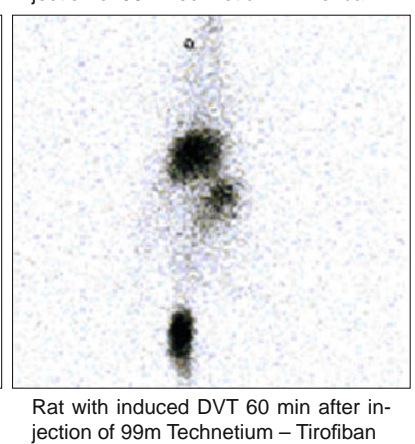

Fig. 5. Gamma camera images after IV injection of 99mTc-tirofiban in rats

In vitro platelet binding

The binding study made for in vitro stability of platelets showed that $99 \mathrm{mTc}$-labeled tirofiban has a high percentage of labeling normal platelets from both, humans and rats, namely 69.30 $\%$ and $68.79 \%$, respectively (Fig. 3). The stability of binding did not change after two washing steps, $58.63 \%$ and $57.58 \%$.

Blood clearance

The obtained results showed that more than $80 \%$ of the injected dose from the labeled preparation of tirofiban was eliminated from the circulation in the first hour after injection (Fig. 4).

\section{Imaging studies}

Whole-body images of distribution of 99m-technetium-tirofiban in normal and experimentally induced thrombosis in rats 30 and 60 min after administration are presented in Figure 5. At
30 and 60 min after injection, experimentally induced thrombi are visualized and compared with normal rat distribution of the tracer. The biological data demonstrated 99m-technetium-tirofiban accumulation in the thyroid and liver. This accumulation could be related to the normal distribution of circulated platelets in the liver and spleen.

Biodistribution studies

The biological distribution data for the labeled 99m-technetium-tirofiban at different times after IV administration are presented in Figure 6. These biodistribution studies demonstrated a different behavior. The results obtained from the radioactivity distribution of 99m-technetium-tirofiban at 30 and 60 min after IV administration clearly present an accumulation in critical organs. This is in accordance with the presence of destroyed platelets carrying the radioactive tirofiban. However, the radiolabeled product was rapidly cleared from the circulation as represented by the low percentage of radioactivity in the heart and lung. The obtained ratio of 'left leg positive for DVT' to 'right leg negative for DVT' was 1.54 after $30 \mathrm{~min}$ and 5.04 after $60 \mathrm{~min}$ for tirofiban labeled with technetium (Fig. 7). These values were considered positive in the detection of acute DVT.

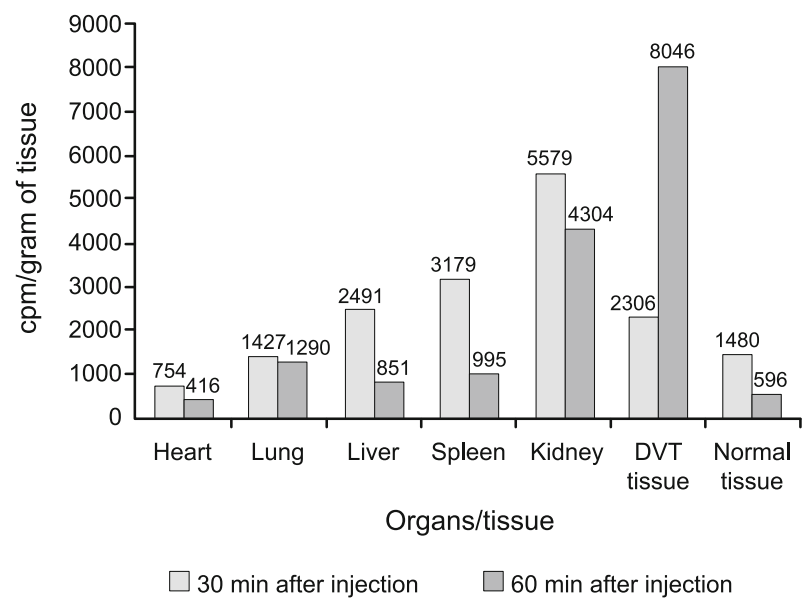

Fig. 6. Biodistribution studies in rat with induced DVT and after injection of $99 \mathrm{~m}$ Technetioum-labeled tirofiban

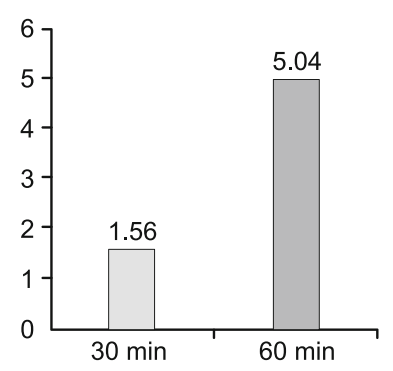

Left thrombotic right normal leg ratio

Fig. 7. Left thrombotic/right normal leg ratio using ROI technique from gamma camera images after injection of 99mTechnetium-tirofiban. 


\section{Discussion}

Blood coagulation has long been considered an important factor in the pathogenesis of venous thrombosis. The relative contribution of stasis, altered coagulability of the blood, vessel wall damage and circulation of leucocytes or platelets to the pathogenesis of venous thrombosis remains in dispute. On the other hand, it is known that a hypercoagulable state contributes significantly to the thrombotic process. Animal models based on these activation mechanisms have been designed as a means of studying potential imaging agents for the diagnosis of acute DVT. The venous stasis thrombosis model consists of inducing blood stasis in the femoral vein of rats after prior injection of a procoagulant. A radionuclide imaging agent that binds to platelets being incorporated into an active thrombus but one which, if not bound, clears rapidly from the blood would have great potential for acute DVT detection.

Platelets expressed from the cell surface of GPIIb/IIIa receptors undergo a conformational change that makes them available for binding with fibrinogen. Cross-linkage of activated platelets by the bivalent fibrinogen molecule to form a hemostatic plug is the primary episode of thrombosis. An imaging agent, ideally labeled with $99 \mathrm{mTc}$ and capable of binding actively and specifically to the GPIIb/IIIa receptor on activated platelets would give images of active or acute venous thrombosis.

The aim of the study was to evaluate tirofiban as a specific imaging agent to GPIIb/IIIa receptors in a case of experimentally induced acute DVT in a rat experimental model. Radionuclide imaging offers considerable potential for a successful diagnostic agent which would address a few important criteria for rapid, noninvasive, cost-effective and accurate diagnosis. The published data $(7,13,19)$ indicate that radiolabeled tirofiban binds preferentially to GPIIb/IIIa receptors on activated platelets and can differentiate acute DVT from chronic venous thrombosis. Radiolabeled tirofiban is a functional rather than anatomical imaging modality. The use of radiotracers allows an understanding of the bioavailability process, biodistribution and kinetics of any molecule labelled with an isotope, a procedure which does not alter the molecule's biological properties. In the current work, technetium (to evaluate tirofiban as a specific imaging agent to GPIIb/IIIa receptor in the case of experimentally induced acute DVT in the rat experimental model) was chosen as a radiotracer for biodistribution and imaging studies in the experimental thrombosis induced in rats for its favourable radiation and physical characteristics, ready availability, possibility of labelling and low cost.

Commercially available technetium-apcitide (AcuTect), previously known as 99mTc-P280, which binds to the GPIIb/IIIa receptor, is the first imaging agent used in the clinical studies to detect acute DVT $(21,22)$. In this study, although the $99 \mathrm{mTc}$-apcitide images obtained at $2 \mathrm{~h}$ after tracer injection show the greatest overall accuracy in comparison with earlier images, a combined analysis of image sets from at least two time points (30 min and $60 \mathrm{~min}$ ) provides greater accuracy in the detection of acute DVT in the patients. These results correlate with data obtained from the animal model. The $99 \mathrm{~m}$-technetium-tirofiban is accurate in the detection of acute DVT, especially 60 min after application. The results obtained from the animal design experimental studies showed that the ratios of 'left leg positive for DVT' to 'right leg negative for DVT' for the radiolabelled preparation of tirofiban are well within the range of that expected from a successful imaging agent.

\section{Conclusion}

Animal models provide convenient screening tools for radiolabeled products before a new radiopharmaceutical is further developed in clinical trials (23).

The tirofiban labelled with 99m-technetium was found to have good thrombus uptake in vivo. Obtained data indicate that radiolabeled tirofiban binds preferentially to GPIIb/IIIa receptors on activated platelets and can differentiate acute DVT from chronic venous thrombosis.

These results can be helpful in further clinical investigation of patients with acute DVT.

\section{References}

1. Hyers TM. Venous thromboembolism. Am J Resp Crit Care Med 1999; 159: 1-14.

2. Horlander KT, Mannino DM, Leeper KV. Pulmonary mortality in the United States, 1979-1998. Arch Intern Med 2003; 163: 1711-1721.

3. Medicure Pharma. Aggrastat (Tirofiban hydrochloride) injection premixed and injection prescribing information. Somerset, NJ; 2007 Nov.

4. Tirofiban hydrochloride. Drugs Future 1995; 20: 897-901.

5. Aruva MR, Daviau J, Sharma SS, Thakur ML. Imaging Thromboembolism with Fibrin-Avid 99mTc-Peptide: Evaluation in Swine. J Nucl Med 2006; 47 (1): 155-162.

6. Knight LC, Radcliffe R, Maurer AH et al. Thrombus imaging with $99 \mathrm{mTc}$ synthetic peptides based upon the binding domain of a monoclonal antibody to activated platelets. J Nucl Med 1994; 35: 282-288.

7. Knight LC, Maurer AH, Romano JE. Comparison of iodine-123-disintegrins for imaging thrombi and emboli in a canine model. J Nucl Med 1996; 37: 476-482.

8. Pearson DA, Lister-James J, McBride WJ et al. Thrombus imaging using 99mTc labeled high potency GPIIb./IIIa receptor antagonist: chemistry and initial biological studies. J Med Chem 1990; 39: 1372-1382.

9. Lister-James J, Vallabhajosula S, Moyer BR et al. Pre-clinical evaluation of technetium-99m platelet receptor-binding peptide. J Nucl Med 1997; 38: 105-111.

10. Line BR, Cran P, Lazewatsky J et al. Phase I trial of DMP444, a new thrombus imaging agent (abstract). J Nucl Med 1996; 37 (Suppl): 117P.

11. Barrett JA, Damphousse DJ, Heminway SJ et al. Biological evaluation of $99 \mathrm{mTc}$ cyclic glycoprotein IIb/IIIa receptor antagonists in the canine anteriovenous shunt and deep vein thrombosis models: effects of chelators on biological properties of $(99 \mathrm{mTc})$ chelator-peptide conjugates. Bioconjug Chem 1996; 7: 203-208.

12. Coller BS. Cell adhesion in vascular biology: Platelet GPIIb/IIIa antagonists: The first anti-integrin receptor therapeutics. J Clin Invest 1997; 99: $1467-1471$. 


\section{$621-626$}

13. Verstraete M. Synthetic inhibitors of platelet glycoprotein IIb/IIIa in clinical development. Circulation 2000; 102: e76-e80.

14. Weiss DJ et al. Platelet kinetics in dogs treated with a glycoprotein IIb/IIIapeptide antagonist. Toxicol Pathol 2000; 28: 310-316.

15. ICH Topic Q 2 (R1) Note for guidance on validation of analytical procedures: Text and methodology (CPMP/ICH/381/95), European Medicines Agency, London, 1995.

16. Ranjitha KS, Rao AL. Development and validation of new RP-HPLC method for determination of Tirofiban in pharmaceutical formulation. IJPCBC 2011; 1 (1): 43-47.

17. http://www.accessdata.fda.gov/drugsatfda_docs/label/2012/020912s0 $18,020913 \mathrm{~s} 017 \mathrm{lbl}$.pdfassessed on $14^{\text {th }}$ of July, 2012.

18. Callas JW, Fareed DDJ. A survey of animal models to develop new and novel antithrombotic agents. In: Sasahara AA, Loscalzo JL (Eds). New Therapeutic Agents in Thrombosis and Thrombolysis 1997; 9-28.

19. Herbert JM, Bernat A, Maffrand JP. Importance of platelets in experimental venous thrombosis in the rat. Blood 1992; 80: (9): 2281-2286.
20. Lister-James J, Mauer A. Thrombus imaging with a technetium 99mlabeled, activated platelet receptor binding peptide. J Nucl Med 1996; 213: 207.

21. Olfert ED, Cross BM, McWilliam AA (Eds). Guide to the care and use of experimental animals. Canad Council Anim Care 1993; 1.

22. Stassen JM. Animal model to assess the safety and efficacy of thrombolytic agents. New Therapeutic Agents in Thrombosis and Thrombolysis. In: Sasahara AA, Loscalzo JL (Eds). New Therapeutic Agents in Thrombosis and Thrombolysis 1997; 451-474.

23. Zhou Y, Chakraborty S, Liu S. Radiolabeled Cyclic RGD Peptides as Radiotracers for Imaging Tumors and Thrombosis by SPECT. Theranostics 2011; 1: 58-82.

24. Carretta RF, Streek PV, Weiland FL. Optimizing Images of Acute Deep-Vein Thrombosis Using Technetium-99m-Apcitide. J Nucl Med Techn 1999; 27: 271-275.

25. Houshmand S, Salavati1 A, Hess S, Ravina M, Alavi A. The role of molecular imaging in diagnosis of deep vein thrombosis. Am J Nucl Med Mol Imaging 2014; 4 (5): 406-425. 